\title{
Le Tableau numérique et organisation scolaire : une enquête vidéo-ethnographique
}

\section{Dimitri Voilmy}

\section{(2) OpenEdition \\ 12 Journals}

Édition électronique

URL : http://journals.openedition.org/communicationorganisation/4249

DOI : 10.4000/communicationorganisation.4249

ISSN : $1775-3546$

Éditeur

Presses universitaires de Bordeaux

\section{Édition imprimée}

Date de publication : 1 juin 2013

Pagination : 255-268

ISBN : 978-2-86781-883-7

ISSN : $1168-5549$

\section{Référence électronique}

Dimitri Voilmy, «Le Tableau numérique et organisation scolaire : une enquête vidéo-ethnographique », Communication et organisation [En ligne], 43 | 2013, mis en ligne le 01 juin 2015, consulté le 19 avril 2019. URL : http://journals.openedition.org/communicationorganisation/4249; DOI : 10.4000/ communicationorganisation. 4249 


\title{
Le Tableau numérique et organisation scolaire : une enquête vidéo-ethnographique
}

\author{
Dimitri Voilmụ ${ }^{1}$
}

Le dispositif du tableau numérique comporte un tableau blanc augmenté électroniquement, relié à la fois à un ordinateur et à un vidéo projecteur. Il suffit de toucher l'écran tactile avec le doigt ou le stylet pour contrôler les applications et annoter les documents. Au quotidien, l'ordinateur est intégré à l'organisation de l'apprentissage dans la salle ; ce n'est plus une relation personnelle que l'élève entretient avec un PC. Le tableau numérique est un objet de la vision novatrice de l'informatique ubiquitaire (Ishii, 2004 : 1299 ; Weiser, 1991). On le sait, le tableau numérique combine la surface pour les visualisations avec les capacités des applications d'un ordinateur. En venant au tableau, les élèves manipulent des éléments visuels par le clic informatique et en les faisant glisser/déposer sur l'écran. De manière grandissante, plutôt qu'écrire au tableau en tournant le dos aux autres, des tablettes mobiles sont utilisées pour écrire à distance. La question de recherche est : est-ce que l'arrivée d'une nouvelle technologie affecte les formes organisationnelles des cours et transforment les pratiques de travail et de communication ? Retrouve-t-on une différence notable dans les pratiques d'enseignement selon que l'enseignement est supporté par un tableau traditionnel ou un tableau numérique ? Les compétences interactionnelles que mobilisent les acteurs en situation de classe s'en trouvent-elles modifiées par un support d'inscription « interactif », où l'interaction est à la fois entre les personnes et avec le tableau ? Il s'agit ici d'une problématique nouvelle, tout autant technologique que sociale, à laquelle les recherches commencent juste à s'intéresser. Cet article interroge l'usage du tableau numérique en classe comme un cas très

1 Dimitri Voilmy est docteur en sociologie de Télécom ParisTech. Ses recherches sont basées sur des enregistrements en vidéo d'activités sociales de la vie quotidienne. Elles intègrent le rapport entre parole et regard pour l'organisation de l'action et la coopération, ainsi que la communication médiée par des visualisations. Dans ce cadre, il s'intéresse à l'attention conjointe comme phénomène social observable dans les champs des IHM et de l'informatique ubiquitaire ; dimitri.voilmy@unice.fr 
particulier de foyer interactionnel qui implique l'interaction entre l'humain et la machine, et participe de l'organisation de l'interaction entre les personnes.

\section{Usages du tableau numérique pour la coopération}

L'étude longitudinale sur la façon dont se déroulent les réunions de travail informelles a montré que les personnes laissent souvent des dessins ou des notes sur le tableau (Ju et al., 2007). Dans les groupes de travail, ces notes sont laissées pour fournir une référence persistante sur le thème qui a été discuté. En cela, il est intéressant d'étudier la mise en correspondance oral/écrit en relation avec la structuration globale des situations de coopération autour des tableaux. En effet, l'une des fonctions principales de l'écriture (Goody, 1979 : 145) est le stockage de l'information. Elle « permet de communiquer à travers le temps et l'espace et fournit à l'homme un procédé de marquage, de mémorisation et d'enregistrement "(ibid.). L'écriture assure également «le passage du domaine auditif au domaine visuel » (ibid.), permettant de réarranger ou de rectifier des phrases. Le stockage des informations du tableau, tout autant que leur partage deviennent les ressources des plateformes réseaux de service pour la communication et l'organisation.

Les études (Bly, 1988 ; Streitz et al., 1994 : 347 ; Stafford-Fraser et Robinson, 1996) qui ont exploré les avantages et inconvénients de tableaux d'affichage et d'écriture dans les bureaux les ont abordés d'une perspective technologique. Elles ont retenu trois faiblesses majeures à l'utilisation du tableau traditionnel $:$ i) trouver un espace utilisable parmi les contenus déjà utilisés et qui ne doivent pas être effacés, ii) la difficulté de partager de l'information à la suite de la discussion, iii) les éléments, une fois effacés, ne peuvent pas être récupérés. Or, les tableaux numériques permettent précisément de transformer ces caractéristiques afin d'afficher du contenu informatique, de modifier, de sauver et d'imprimer les informations affichées. Le premier tableau augmenté électroniquement était le LiveBoard développé à Xerox PARC en 1987. Il a connu quelques modifications matérielles pour se stabiliser en 1992 (Buxton, 1997). En parallèle, la société canadienne SMART Technologies lance la première commercialisation d'un tableau numérique avec écran LCD en 1991 avec son SMART Board. Ensuite, Tivoli, également conçu par Xerox, fonctionnait à partir de la base matérielle du LiveBoard (Pedersen et al., 1993). Celui-ci avait été installé dans un petit bureau pour les réunions de travail. On trouve également ces dispositifs dans un bureau individuel (Mynatt et al., 1999), et comme outil de collaboration à distance (Elrod et al., 1992), par exemple pour faire correspondre en simultané les écrits de deux participants distants (Ishii et al., 1994 : 83). En effet, le tableau numérique autorise des applications partagées pour des opérations collaboratives en ligne.

$\mathrm{Du}$ fait de ces caractéristiques, le tableau numérique comporte des bénéfices certains pour la situation d'enseignement, bien que, nous le verrons, 
il ne modifie pas fondamentalement les pratiques organisationnelles dans l'éducation. Il combine les deux innovations principales de l'informatique : son environnement graphique avec l'interface utilisateur et l'extension de son champ d'action avec l'Internet. Il permet de mettre au même emplacement et de manière simultanée, de nombreux contenus pédagogiques : l'accès aux applications liées à l'internet et aux ressources numériques (graphiques, textes, dessins, matériel pédagogique, le travail étudiant, vidéo, audio, cartes, films, jeux, encyclopédies, dictionnaires, reportages TV). Les enseignants découvrent le choix important d'applications logicielles avec des ressources pédagogiques et des contenus avec les médias sociaux présents sur le web. Des opérations liées à l'informatique en réseau sont introduites à l'intérieur de la salle de classe. Les apprenants sont réceptifs et actifs. Formant des communautés en ligne, ils participent aux réseaux sociaux qui ont réinventé la société et accroissent la portée et les usages de la technologie Internet (Castell, 2001 : 79), dans une continuité de l'ancrage de l'informatique dans les pratiques scolaires et le contexte pédagogique local. Néanmoins, l'analyse de l'usage scolaire du tableau numérique ne se limite pas à son aspect technologique.

Les pratiques réelles dans lesquelles les apprenants et les enseignants sont engagés, et le sens que peuvent avoir pour eux de telles pratiques, nous informent sur les usagers de l'informatique en éducation (Baron et Bruillard, 1996). La recherche sur les communications par ordinateur se développe dans une orientation plus écologique, en considérant la participation des apprenants, qui rassemble des formes d'engagement mutuel et d'activités conjointes (Crook, 2009). L'organisation locale de l'interaction en classe est accomplie par des processus de compréhensions verbales et de gestuelles corporelles. À cet égard, les ressources qu'utilisent les participants en situation de face-à-face peuvent être des objets et des documents, mais aussi des instruments, des outils, ou des technologies. Goffman (1987: 45) observe que « la structure même du contact social met en jeu des mouvements matériels » lesquels s'ajustent avec la parole dans des phases d'action. C'est pour cette raison que mon intérêt pour les supports des visualisations ne saurait dissocier l'aspect technologique du tableau numérique de l'aspect social, avec les manières de faire ordinaires (de Certeau, 1980) avec ce support de l'organisation de l'événement de classe. La prise en compte des objets ouvre, non seulement, un champ plus large que l'analyse du travail pédagogique et éducatif, mais elle introduit aussi l'étude des tâches quotidiennes avec le matériel destiné à certaines activités et avec des interfaces des technologies informationnelles (Conein, 1997 : 26).

La littérature sur l'éducation a montré que le tableau - qu'il soit traditionnel ou numérique ${ }^{2}$ - tient une place centrale dans la structuration de l'interaction pédagogique (Macbeth, 2000 ; Greiffenhagen, 2000 ; Nolan, 2008). C'est autour de ces surfaces pour les visualisations que s'organise l'action sociale

2 Il faut noter que le tableau numérique vient s'ajouter au matériel de la classe et non se substituer à l'existant. 
dans la salle de classe, permettant l'attention conjointe, qui est essentielle au succès de la communication. Selon Tomasello (1995 : 107), le point central est que l'attention conjointe ne signifie pas seulement deux personnes regardant la même chose au même moment. Ce n'est pas juste une personne spectatrice tandis que l'autre s'engage avec un objet ; ce n'est pas l'élève alternant son attention entre deux phénomènes d'intérêt égal (une personne et un objet). Dans l'attention conjointe, l'élève $\mathrm{A}$ coordonne son attention à l'objet et à l'élève $B$ en même temps que l'élève $B$ coordonne son attention au même objet et à l'élève $\mathrm{A}$. Cet arrangement spatial, et l'action qu'il soutient, a permis d'interroger le lien entre organisation des activités et environnement selon l'intérêt des nouvelles sociologies (Conein, 1997). M'inspirant de cette approche, l'information ethnographique fournit l'arrière-plan dans lequel l'analyse vidéo est réalisée, alors que la compréhension détaillée fournie par l'analyse-micro de l'interaction, à son tour, informe notre compréhension générale ethnographique (Jordan et Henderson, 1995). En privilégiant l'organisation de l'action dans le fil d'une sociologie qualitative moderne de l'éducation (Coulon, 1989 : 124), Hugh Mehan (1979) analyse l'organisation de séquences interactionnelles entre enseignants et élèves à partir d'extraits collectés en classes d'écoles primaires. Dans le prolongement de ses travaux, mon dispositif de captation audio et vidéo permet d'évaluer les visualisations et l'usage de matériels physiques (des tableaux de cours en particulier) pour la coordination de l'action et la " compétence interactionnelle » (Mehan 1979) que les élèves exposent de manière publique.

\section{Agir avec le tableau numérique}

En démarrant mon ethnographie vidéo, j’ai observé des usages innovants dès l'école primaire. Fait nouveau, et usage grandissant, les élèves apprennent à faire fonctionner des dispositifs périphériques sans fil pour un usage à distance avec la surface du tableau. Weiser (1998 : 42) analysait très justement que « la communication sans fil sera la clé pour lier ensemble les diverses fonctions des ordinateurs autour de nous, et un environnement de campus compact le rend plus facile ». C'est cette manipulation du tableau numérique à partir d'une tablette mobile qui sera discutée ici. Avec le photomontage de différentes vues vidéo de la figure ci-dessous, il est possible de voir l'activité qui est réalisée à distance par l'élève assis avec les autres élèves. De nombreuses informations, tel un inventaire des rubriques du calendrier, sont affichées au tableau : la liste des jours de la semaine, les mois de l'année, les saisons et la météo.

La consigne de l'exercice est affichée au tableau. L'enseignante interroge un élève pour lui demander quel était le jour précédent. Lélève construit une nouvelle phrase avec la réponse qu'il a donnée. Il manipule un stylet électronique pour déplacer les zones de texte et remplir les champs laissés vides par l'enseignante. La tablette portable permet une utilisation dans tout le périmètre de la pièce. Tout ce que l'élève écrit/fait sur la tablette est projeté sur 


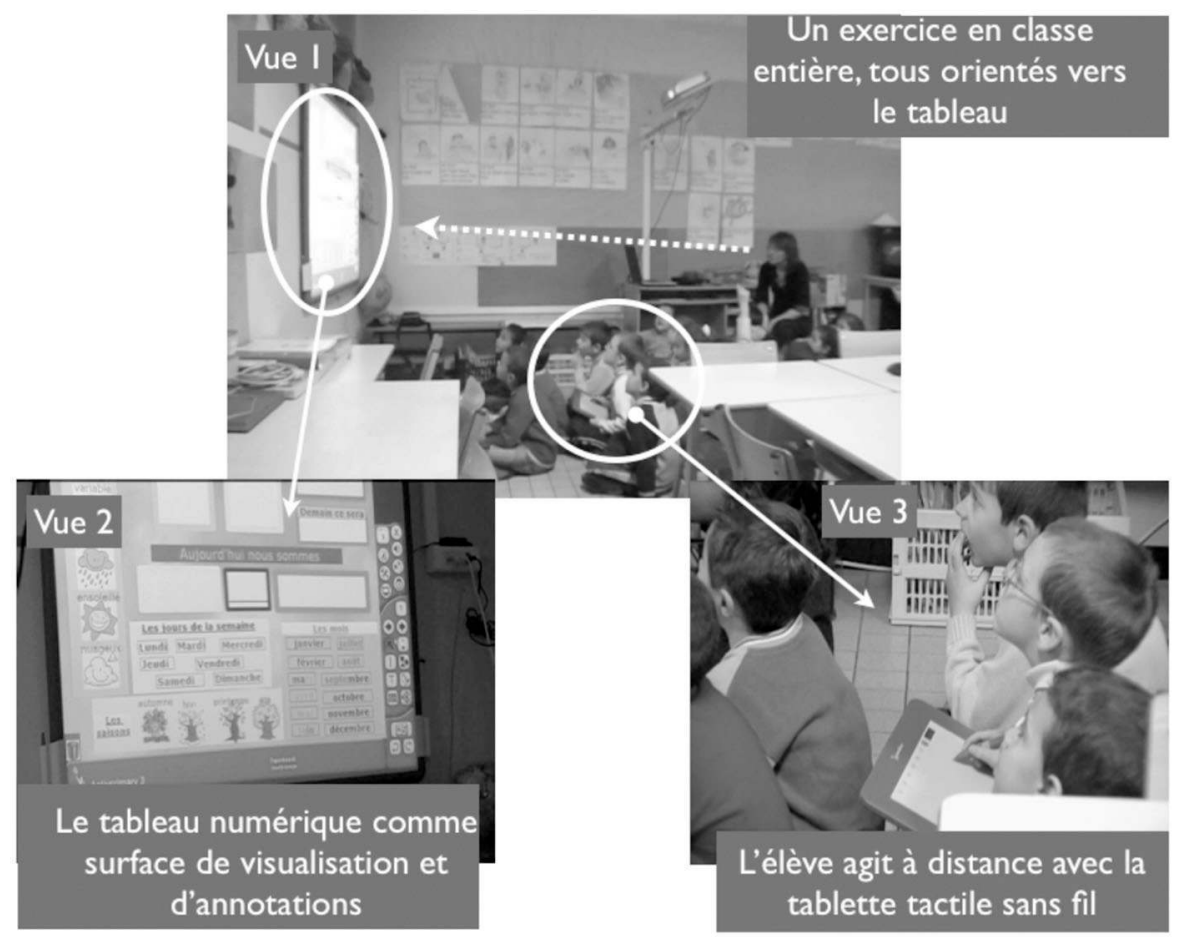

Figure 1 : Équipement de tablette tactile sans fil pour l'exercice au tableau numérique, filmé à Grasse en novembre 2007

le grand écran, ce qui permet l'observabilité (et un contrôle) par l'enseignant et les élèves. Dans cette classe de cours préparatoire, l'enseignante assise sur une chaise fait la leçon en gardant une vue sur le tableau et ses élèves. Dans la configuration en classe entière, comme c'est le cas ici, l'écriture au tableau est un travail de classe en équipe. La tablette tactile est transmise d'un élève à l'autre. Les élèves sont assis face au tableau, et leur proximité favorise les échanges, comme lorsqu'un élève techniquement plus en confiance donne des conseils sur la façon de tenir le stylet. Sur ce thème, en Angleterre, Charles Crook de l'Université de Nottingham étudie les formes de l'attention visuelle chez les enfants, associées à la fluidité de production de l'écriture. Aussi, il montre que la coordination de l'activité d'écriture médiée par un outil présente des défis pour le développement de ces artefacts. Pour cela, il suggère d'aider les enfants vers plus de confiance du contrôle visio-manuel des outils d'écriture (Crook, 2009).

On observe ici les nouvelles dispositions apportées à la leçon, qui n'étaient pas disponibles avec des outils tels que le livre ou la projection sur un écran d'un document statique. La différence principale du tableau numérique d'avec un tableau traditionnel est précisément que le contenu n'est pas «statique ", 
mais comporte des caractéristiques réactives propres à l'artefact informatique interactif. Selon la définition de Suchman (2007), l'artefact informatique interactif permet un accès mutuel à la surface pour les visualisations - qui contient les annotations, les traces d'actions, les résultats d'erreurs de manipulation. Contrairement à un affichage préalablement écrit, un tableau donne accès non seulement au produit écrit, mais au processus de production de l'écrit - un processus, qui plus est, coopératif, du fait de la participation des autres élèves. L'avantage de l'utilisation de la tablette mobile est que les participants peuvent se voir, en face-à-face (Fig. 2) ou côte-à-côte (Fig. 1). Écrire de manière à rendre son écrit visible pour les autres n'implique plus de se mettre dans une posture - face au tableau et dos aux participants - où l'interaction interpersonnelle serait entravée. Par conséquent, il en résulte une plus grande co-participation. " Passer chacun son tour » au tableau n'est pas une activité individuelle de chaque élève, mais une activité de coopération, où les élèves s'entre-aident en temps réel et apprennent à travailler ensemble (Voilmy, 2009).

L'appareil périphérique contrôlant le tableau peut également être posé sur un bureau, dans lequel cas, les élèves sélectionnés pour l'exercice se déplacent chacun leur tour. Ci-dessous, on peut voir la disposition pratique d'une classe d'économie-gestion avec des lycéens, dans laquelle l'enseignant utilise sa tablette PC personnelle pour un apprentissage collaboratif.

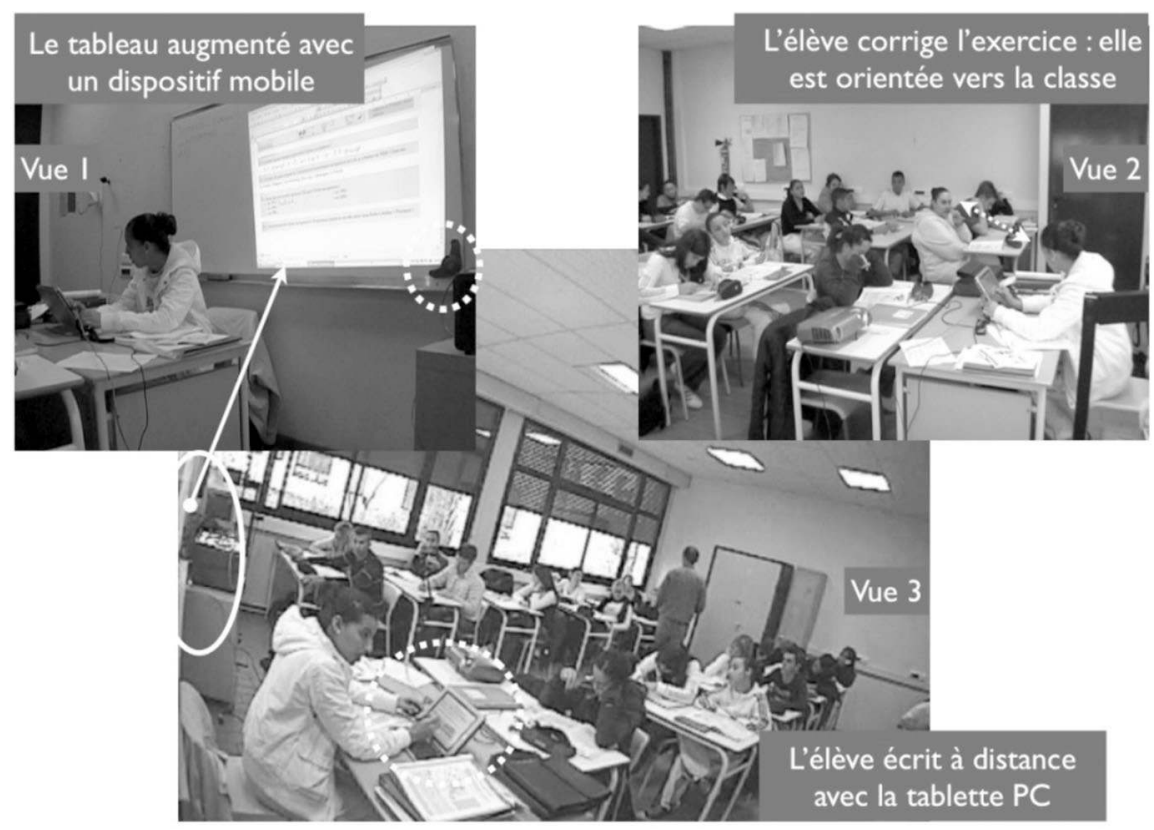

Figure 2 : Équipement de tablette PC au bureau de l'enseignant pour corriger l'exercice au tableau numérique, filmé à Cavaillon en mai 2007 
Le cours porte sur la construction et l'organisation de l'Union européenne. L'enseignant demande d'écrire les listes des pays qui ont rejoint l'union européenne en 1973, 1981, 1986, 1995, 2004 et 2007. Dans un premier temps, les élèves répondent aux questions sur leur feuille. Puis pour la correction, une élève au tableau réécrit les bonnes réponses en remplissant le document numérique. Chacun peut voir la composition de sa réponse en même temps qu'elle l'écrit. Une élève assise dans la salle lui donne une indication qu'elle prend en compte pour compléter l'écriture de sa réponse. Linstallation matérielle de l'environnement technologisé dans ces classes présente des ressources qui facilitent la collaboration entre les élèves et l'attention conjointe vers ce qui est en train d'être écrit. Aussi, les retours que j'ai du terrain, sont que les élèves sont favorables au travail avec un tableau numérique en classe. Dans ce sens, ils demandent explicitement à ce que le contenu du cours puisse être enregistré en multimédia, pour « la possibilité de pouvoir rejouer le cours en disposant de l'ensemble des contenus communiqués par l'enseignant » (Lecolinet et al., 2005). L'activité d'annotation fait partie de l'exposé et de la réalisation du cours. L'enregistrement des annotations, en prenant des captures d'écran avec un enregistrement audio par exemple, implique l'usage d'un tableau numérique. En observant ces usages ordinaires du tableau en classe, il est possible d'améliorer des fonctionnalités existantes à la fois matérielles et logicielles. L'équipe de Lecolinet (2005) a mis en place des solutions pour que les annotations du cours soient partagées en temps réel avec les récepteurs des élèves via un réseau sans fil. Cette propriété peut permettre une forme de travail collaboratif synchrone de plusieurs participants, chacun voyant l'application affichée sur sa propre machine. La communication multilatérale et synchrone est disponible entre le tableau numérique et un récepteur mobile, ce qui rend disponible un niveau de contrôle par les parties en interaction. Ainsi, le niveau de l'interactivité correspond à l'étendue du contrôle exercé par les parties en interaction (Van Dijk, 2006 : 8).

\section{Visualisations partagées et « interaction "}

D'une perspective émique (Pike, 1967), c'est-à-dire selon le point de vue et les termes des acteurs observés, ces outils sont couramment appelés «tableaux interactifs ». Les commandes au tableau exécutées par la personne sur la surface tangible, avec un stylet ou au doigt, sont des représentations d'objets qui se comportent comme s'ils étaient les objets eux-mêmes, offrant le sentiment d'immédiateté de la manipulation (Shneiderman, 1982). L'aspect d'immédiateté n'est pas une propriété de l'interface seule. L'engagement direct implique une relation entre la tâche que l'utilisateur souhaite effectuer et la manière dont cette tâche peut être accomplie par l'intermédiaire de l'interface (Hutchins, Hollan, Norman, 1985). Le terme « interaction » est utilisé en interaction homme-machine pour caractériser également ce qui se passe entre des personnes et certaines machines. Un programme informatique 
est dit « interactif » si celui-ci est conçu pour demander des informations à l'utilisateur. Or, ce modèle de l'interaction met en avant la logique des systèmes informatiques au détriment de la co-participation et la coopération. Heath et Vom Lehn (2008) ont analysé l'« interactivité » dans les expositions informatisées de centres des sciences ou de musées. Ils montrent que les expositions informatisées soutiennent une attention prolongée, à la fois vers une tâche ou une activité unique, non pas pour l'ensemble des participants, mais pour un utilisateur individuel : la majorité des visiteurs ne font que regarder l'utilisateur principal « interagir » avec le système.

La description des artefacts informatiques comme interactifs, leur apportant ainsi une sociabilité relative grandissante, est basée sur leurs caractéristiques réactives, linguistiques et intérieurement opaques (Suchman, 2007 : 34-35). En effet, le moyen pour contrôler la machine est de plus en plus linguistique, plutôt que mécanique : au lieu d'appuyer sur des boutons avec un résultat physique, il s'agit de spécifier les opérations et d'évaluer leurs effets à travers l'utilisation d'un langage commun. Ce sont précisément les caractéristiques réactives et linguistiques des tableaux numériques, et les effets des opérations qui nous intéressent, à savoir la manière dont l'écriture sur ce périphérique mobile qu'est la tablette est rendue visible en temps réel sur la surface.

Cependant, il est utile de préciser que l'interactivité de la machine a ses limites, comme l'explique Suchman, et ne suscite pas nécessairement d'activités d'apprentissage (Baron et Bruillard, 1996 : 30ch4). La différence principale entre l'interaction humaine et l'interaction homme-machine est la compétence sociale et interactionnelle des personnes. Les personnes parviennent non seulement à communiquer, mais également à afficher leur compréhension de manière réflexive et à gérer l'observabilité de leur conduite, en tenant compte des contingences et en s'adaptant aux contextes. Ce faisant, elles parviennent à produire l'attention conjointe et à maintenir l'intersubjectivité. Or, dans cette production de l'attention conjointe et de l'organisation de la classe, mes recherches ont démontré que le dispositif pour les visualisations constitue une ressource essentielle. L'affichage en temps réel du contenu du cours soutient l'interaction entre l'agent qui écrit et les coparticipants qui peuvent voir le produit écrit, et suivre l'action. Cependant, contrairement à la situation d' «écrire au tableau »-que celui-ci soit traditionnel ou augmenté - où l'agent se trouve dans une position tourné vers le tableau et collé à lui, où il ne voit pas ses co-participants, la tablette permet une configuration différente. Assis, tourné vers ses co-participants ou assis à côté d'eux, l'agent qui écrit à la tablette, voit ses co-participants et peut interagir plus aisément avec eux. L'agent continue d'« interagir " avec le dispositif numérique, comme il le ferait avec un stylet. Mais nous voyons que la tablette mobile permet une interaction plus « naturelle » avec les autres personnes en vue d'accomplir l'action d'écriture. Dans les deux exemples ci-dessus, nous voyons comment la coopération est accomplie, précisément à travers 
l'accomplissement de l'attention conjointe vers l'activité en cours autour du, et soutenue par, le tableau numérique. L'agent au tableau n'accomplit pas son activité seul, mais avec la participation active des autres "participants " qui, par leur attention à l'action de l'agent au tableau, font davantage que regarder comme une audience. Il apparaît donc un groupe de participants qui suivent et accomplissent ensemble une seule et même activité.

Les connaissances dégagées de situations réelles de pratiques soutenues par les visualisations partagées permettraient d'apporter un certain nombre de réponses aux questions relatives à l'accomplissement social, que les designers ne se posent pas forcément en concevant des outils. La manière dont l'attention conjointe est accomplie en lien avec les objets, notamment les objets informatiques, se révèle utile eu égard aux lacunes pointées par la littérature concernant l'« interactivité » des artefacts informatiques et l'interaction sociale et humaine. Or, les écueils dus aux erreurs dans la conception et qui ont abouti à un résultat décevant, pointés par Heath et Vom Lehn, ont justement été évités dans les extraits d'usage du tableau numérique, que j'ai analysés. Nous nous concentrerons sur deux d'entre eux. Le premier est le modèle de l'utilisateur principal, qui restreint la co-participation et la collaboration. Le second est que, même dans les conceptions où il est possible d'interagir simultanément à plusieurs avec le système, le fait de ne pas avoir accès aux opérations et aux contributions des autres, ne permet pas la coordination et une activité cohérente, qui fait l'objet de l'attention mutuelle des participants. Or, l'on constate, à partir des analyses d'extraits d'événements de classe autour du tableau numérique, que la coordination est effectivement accomplie, par l'attention conjointe. Bien que l'agent qui tient la tablette soit le seul à avoir la main sur les actions à effectuer, son action est loin d'être individuelle. La co-participation est accomplie par le fait de pouvoir suivre les actions de l'agent, par l'accessibilité à la surface pour les visualisations, et également par le fait de pouvoir interagir en face-à-face. Cela démontre ainsi que la dimension sociale et humaine, c'est-à-dire l'« interaction " telle que nous la concevons, ne doit pas être négligée quand on s'intéresse à analyser ou à concevoir l'« interactivité » des artefacts informatiques.

\section{Discussion : Interactions à plusieurs sur tableau numérique}

Les ordinateurs, et plus globalement les technologies informatiques, jouent désormais un rôle majeur dans pratiquement chaque aspect de notre vie quotidienne (Harper et al., 2008). Cela va de l'informatique ambiante au bureau aux TICE pour l'apprentissage à l'école dès le plus jeune âge. Le défi pour les IHM est d'évoluer de la simple conception de meilleurs systèmes informatiques vers une conception qui inclut les valeurs, la morale et l'éthique humaines, c'est-à-dire une conception pas simplement centrée utilisateur, mais centrée sur l'humain. Le modèle de recherche actuel dans la conception centrée utilisateur est un modèle en quatre étapes : Étudier/ 
Concevoir/Construire/Évaluer. Une évolution vers un modèle de conception centrée sur l'humain nécessiterait de rajouter une cinquième étape : Comprendre - comprendre les valeurs humaines et concevoir en fonction d'elles (Harper et al., 2008). Cette nouvelle étape, je la développe par une démarche qui vise à comprendre l'« interaction » avec l'objet qu'est le tableau, comprendre l'ordre social et l'accomplissement interactionnel, comprendre la coproduction continue et contingente d'un monde socio-matériel partagé, qu'on appelle " interaction » (Suchman, 2007), comprendre l'attention conjointe comme un accomplissement pratique et éminemment social. Cette méthode de recherche, tournée vers la compréhension et l'étude de l'action sociale, est actuellement très utilisée dans le design d'outils IHM dans le paysage anglo-saxon, suivant le « tournant vers le social " (Crabtree, 2003).

Le stylet ou l'usage de la tablette mobile permettent l'utilisation du tableau par un agent à la fois. La technologie amenée à se démocratiser est celle du tactile multipoint. Comme les tableaux traditionnels où plusieurs personnes peuvent écrire en même temps avec des craies ou des feutres, un tableau tactile multipoint est conçu pour plus d'un utilisateur à la fois sur le même dispositif. Sachant que ces surfaces pour les visualisations et d'annotation fonctionnent par points de contact simultanés et multiples, comment s'accomplit la coordination des actions avec les autres ? Placés côte-à-côte, quelle est l'accessibilité visuelle des différents agents aux actions en temps réel des autres, et aux affichages sur la surface ? Ces questions concernant l'accomplissement de l'activité en commun se poseront nécessairement, étant donné que l'usage du tableau est une activité sociale. Comprendre cette socialité demandera des développements et des recherches futures, utiles à la conception d'outils IHM, pour comprendre afin d'adapter, au mieux, l'arrivée d'outils technologiques qui soutiendraient les pratiques pédagogiques en occasionnant un minimum de perturbations.

\section{BIBLIOGRAPHIE}

BARON G.-L., BRUILLARD E., L'informatique et ses usagers dans l'éducation, Paris, Presses Universitaires de France, 1996.

BLY S. A., "A use of drawing surfaces in different collaborative settings », CSCW, 88, 1988, p. 250-256.

BUXTON W., " Living in augmented reality : Ubiquitous media and reactive environments ", in K. FINN, A. SELLEN, and S. WILBER, Video mediated communication, Hillsdale, NJ Erlbaum, 1997, p. 363-384.

CASTELLS M., « La culture d'Internet » La galaxie internet, Paris, Fayard, 2001, p. 50-81.

CERTEAU de M., L'invention du quotidien, Paris, UGE, 1980.

CONEIN B., "L'action avec les objets. Un autre visage de l'action située ? ", Raisons Pratiques, 8, 1997, p. 25-46. 
COULON A., " Note critique de l'ouvrage de Hugh Mehan, Alma Hertwerck et J. Lee Meihs "Handicapping the Handicaped" ", Revue française de pédagogie, 87, 1989, p. 120-124.

CRABTREE A., Designing collaborative systems : A practical guide to ethnography, London, Springer-Verlag, 2003.

CROOK C., "Coordinating, collaborating and blending with technology ", in M. B. Ligorio et al. eds., Talking over the computer: pedagogical scenarios to blend computer and face to face interaction, Scriptaweb, 2009, p. 53-64.

ELROD S. et al., " Liveboard: a large interactive display supporting group meetings, presentations, and remote collaboration ", $C H I$ '92, 1992, p. 599-607.

GOFFMAN E., Façon de parler, Paris, Les Editions de minuit, 1987.

GOODY J., La raison graphique : la domestication de la pensée sauvage, Paris, Les Éditions de Minuit, 1979.

GREIFFENHAGEN C., « From traditional blackboards to interactive whiteboards: a pilot study to inform system design ", The 24th international conference Psychology of Mathematics Education, July 23-27, 2, 2000, p. 305-312.

HARPER R., RODDEN T., ROGERS Y., SELLEN A., Being human: Humancomputer interaction in the year 2020, Cambridge, England, Microsoft Research Ltd, 2008.

HEATH C., VOM LEHN D., " Configuring 'Interactivity': Enhancing Engagement in Science Centres and Museums ", Social Studies of Science, 38, 1, 2008, p. 63-91.

HUTCHINS E., HOLLAN J., NORMAN D., " Direct manipulation interfaces », Human-Computer interaction, 1, 1985, p. 311-338.

ISHII H., "Bottles : A transparent interface as a tribute to Mark Weiser », IEICE TRANS INF E SYST, 87, 6, 2004, p. 1299-1311.

ISHII H., KOBAYASHI M., ARITA K., « Iterative design of seamless collaboration media ", Communications of the ACM, 37, 8, 1994, p. 83-97.

JORDAN B., HENDERSON A., "Interaction Analysis: Foundations and Practice », The Journal of the learning sciences, 14, 1, 1995, p. 39-103.

JU W., LEE B. A., KLEMMER S., « Range: Exploring proxemics in collaborative whiteboard interaction », CHI'2007, 2007, p. 2483-2488.

LECOLINET E. et al., "Augmentation de cours et de réunions dans un campus », UBIMOB'05, 2005, p. 161-168.

MACBETH D., "Classrooms as installations: Direct instruction in the early grades», in H. STEPHEN, F. DAVID, Local educational order, Amsterdam, Philadelphia, John Benjamins publishing compagny, 2000, p. 21-71.

MEHAN H., Learning lessons: social organization in the classroom, Cambridge, Harvard University Press, 1979.

MYNATTE. et al., " Flatland: New dimensions in office whiteboards ", CHI'99, 1999, p. 346-353.

NOLAN K K., "SMARTer music teaching: Interactive whiteboard use in music classrooms ", General Music Today, 22, 2009, p. 3-11.

PEDERSEN E. R., MCCALL K., MORAN T. P., HALASZ F., « Tivoli: an electronic whiteboard for informal workgroup meetings ", CHI'93, 1993, p. 391-398. 
PIKE K., " Etic and emic standpoints for the description of behavior ", Language and thought: an enduring problem in psychology, 1967, p. 32-39.

SHNEIDERMAN B., "The futur of interactive systems and the emergence of direct manipulation ", Behavior and Information Technology, 1, 1982, p. 237-256.

STAFFORD-FRASER Q., ROBINSON P., " BrightBoard: A video-augmented environment », CHI'96, April 13-18, 1996, p. 134-141.

STREITZ N. A., GEIBLER J., HAAKE J., HOL J., « DOLPHIN: Integrated meeting support across local and remote desktop environments and liveboards ", CSCW'94, 1994, p. 345-358.

SUCHMAN L., Human-Machine reconfigurations. Plans and situated actions, 2nd Edition, New York, Cambridge University Press, 2007.

TOMASELlO M., "Joint Attention as Social Cognition ", in C. MOORE, P.J. DUNHAM, Joint Attention: Its Origins and Role in Development, Hillsdale, Lawrence Erlbaum Associates, 1995, p. 103-130.

VAN DIJK J., The network society: social aspects of new media.,London, Sage, 2006.

VOILMY D., "Apprendre à passer au tableau. Organiser collectivement son tour au tableau augmente », ELAH'09, 2009, p. 165-172.

WEISER M., "The computer for the 21st century », Scientific American, 263, 3, 1991, p. 94-104.

WEISER M., "The future of ubiquitous computing on campus ", Communications of the ACM, 41, 1, 1998 p. 41-42.

Résumé : Cet article discute de l'importance de l'interface entre l'attention conjointe et les visualisations partagées. Il interroge, pour cela, l'arrivée du tableau numérique dans la classe. Le tableau numérique, qui combine la surface pour les visualisations avec les capacités des applications d'un ordinateur, est un objet de la vision novatrice de l'informatique ubiquitaire (Weiser, 1991). De manière grandissante, plutôt que d'écrire au tableau en tournant le dos aux autres, des tablettes mobiles sont utilisées pour écrire à distance. Bien que l'élève qui tient la tablette soit le seul à avoir la main sur les actions à effectuer, son action est loin d'être individuelle. À partir des analyses d'extraits en vidéo d'événements de classe autour du tableau, l'on constate l'importance de l'attention conjointe et de quelle manière la coordination est effectivement accomplie. Elle est accomplie par le fait de pouvoir suivre les actions de l'élève, par l'accessibilité aux visualisations, et également par le fait de pouvoir interagir en face-à-face. Cela démontre ainsi que la dimension sociale et humaine, c'est-à-dire l'« interaction ", ne doit pas être négligée quand on s’intéresse à analyser ou à concevoir « l'interactivité » des technologies informatiques.

Mots-clés : ethnographie vidéo, tableau numérique, tablette, conception, interaction, nouvelles technologies pour l'enseignement.

Abstract : This paper discusses the importance of the interface between joint attention and shared visualizations. It questions the arrival of the interactive whiteboard in the classroom. The interactive whiteboard combines the surface for visualizations applications with the capabilities 
of a computer. It is an object of the innovative vision of ubiquitous computing (Weiser, 1991). More and more, instead of writing on the board with one's back turned, mobile tablets are used to write at a distance. Although the student holding the tablet is the only one who can act on the board, his action is by no means personal. Based on the analysis of video extracts of classroom events around the board, we can see the importance of joint attention and how coordination is actually accomplished. It is accomplished by the fact that all the participants are able to follow the student's actions, to have access to the visualizations, and the possibility of interacting faceto-face. This demonstrates that the social and human dimension, that is «interaction», should not be neglected when one is interested in analyzing or designing the «interactivity» of computer technologies.

Keywords : Video-ethnography, Interactive whiteboard, Tablet, Design, Interaction, New Information technologies for education. 
